\title{
EGU21-4707
}

https://doi.org/10.5194/egusphere-egu21-4707

EGU General Assembly 2021

(c) Author(s) 2021. This work is distributed under

the Creative Commons Attribution 4.0 License.

\section{GNSS Interferometric Reflectometry for Station Location Suitability Analysis}

\author{
Jeffrey Verbeurgt ${ }^{1,2}$, Ellen Van De Vijver ${ }^{3}$, Cornelis Stal ${ }^{4}$, and Alain De Wulf ${ }^{1}$ \\ ${ }^{1}$ Department of Geography, Ghent University, Ghent, Belgium \\ ${ }^{2}$ National Geographic Institute, Brussels, Belgium (jeffrey.verbeurgt@ngi.be) \\ ${ }^{3}$ Department of Environment, Ghent University, Ghent, Belgium \\ ${ }^{4}$ Center for Applied Data Science, University College Ghent, Ghent, Belgium
}

National geodetic reference systems can be continuously monitored using applications of Global Navigation Satellite Systems (GNSS). Within these reference systems, Continuously Operating GNSS Reference Stations (CORSs) are often employed to provide 24/7 satellite tracking data. Understanding the influence of the surroundings of a CORS on the recorded satellite tracking data is indispensable for quality analysis of both acquired data and station location suitability. One of the main sources of inaccurate tracking data is the result of the combined reception of direct as well as indirect, environment-reflected satellite signals by the CORS, in which the latter can be considered interference compromising the signal's accuracy. The magnitude of this interference is usually evaluated by the Signal-to-Noise Ratio (SNR), a parameter stored by default in the RINEX interchange format for raw GNSS data. The technique of GNSS Interferometric Reflectometry (GNSS-IR) exploits the availability of the SNR data and has been frequently used for applications such as soil moisture monitoring, detection of vegetation water content, measuring snowfall or determining water levels. In this research, we propose to employ GNSS-IR to investigate the effect of the surrounding on a CORS in order to evaluate station location suitability. More specifically, this will be done by using the signal to estimate the Reflector Height $(\mathrm{RH})$, which depends on the reflector roughness (i.e. the roughness of the surface surrounding the CORS). The quality of this estimation will be validated by comparing with the actual measurement of the RH of the CORS on site.

In our approach, a statistically sound method is developed quantifying the stability of the $\mathrm{RH}$ determination. The proposed methodology consists of using Lomb-Scargle periodograms to select the dominant oscillation frequency of each satellite track SNR data, followed by an analysis and filtering of the peak amplitudes. This leads to the analysis product: number of significant peak amplitudes for an individual CORS over (sub-)daily timeframes. With historical data covering long time periods, statistical analysis of the (sub-)daily timeseries allows for reviewing the station location suitability. In Belgium, CORS are located on two typical positions: in Flanders, the 32 antennas are mainly installed on rooftops of buildings; in Wallonia, the 23 antennas are installed on a concrete pole next to highways. There is no evidence of one choice of station position being more suitable than the other. However, cars are known to be an important factor in signal 
reflections. In our analysis of station suitability, the effect of cars passing by on the highway near a Walloon CORS, but also movements on, e.g., parking lots next to buildings with a rooftop CORS, will be investigated. With the developed methodology, guidelines for station location selection could be further developed, together with a system to continuously monitor CORS position suitability using GNSS-IR, triggering a warning when significant changes in the environment changes the local reflectometry fingerprint. 